\title{
23 SOME CHALLENGES FACING VIRTUALLY COLOCATED TEAMS
}

\author{
Gloria Mark \\ University of California, Irvine \\ U.S.A.
}

\begin{abstract}
Numerous challenges face geographically distributed teams who are expected to perform as physically colocated teams: to provide deliverables, meet project schedules, and to generate feasible and even innovative problem solutions-all from a distance. Limitations due to technology and distance make it difficult for geographically distributed teams to develop necessary social processes in order to function as a "well-formed" team. This paper discusses three challenges for these teams, based on results of an empirical study of virtually colocated teams, along with a reexamination of past empirical studies. These challenges are: (1) achieving a high standard of participation, which affects impressions, interaction patterns, and trust; (2) developing an appropriate culture, for motivation and cooperation; and (3) integrating the remote team suitably into members' local working spheres. In the conclusion, future research directions and technology requirements to help meet these challenges are discussed.
\end{abstract}

\section{Introduction}

This paper is concerned with the relatively new phenomenon emerging in organizations where geographically distributed people meet together and collaborate as a team. The term "virtually colocated team" is becoming increasingly more visible as a research topic due to recent trends of corporate mergers, global markets, and interdisciplinary teamwork. Different technologies are being investigated as to their potential to support real-time

The original version of this chapter was revised: The copyright line was incorrect. This has been corrected. The Erratum to this chapter is available at DOI: 10.1007/978-0-387-35505-4_33 
communication and interaction, as well as asynchronous collaboration in such teams. Some technologies investigated in business settings, which have highlighted the problems and benefits for teams, include: desktop conferencing (Mark, Grudin, and Poltrock 1999), e-mail, telephone, and document exchange (e.g., Sproull and Kiesler 1991; Zack 1993); chat (Bradner, Kellogg, and Erickson 1999; Fitzpatrick et al. 1999); and an audio-only media space (Ackerman et al. 1997).

The goal of this paper is to describe challenges facing geographically distributed teams with long-term agendas who use these technologies. The challenges are derived based on an empirical study, together with a reexamination of past empirical studies. Numerous challenges face these teams, who are expected to perform as physically colocated teams: to provide deliverables, meet project schedules, and generate feasible and even innovative problem solutions. And yet all this must be done at a distance. Team members stem from different departments, organizations, countries, and sometimes even different companies. Sometimes teams meet face-to-face on a regular basis, sometimes rarely, often not at all. How can team members be expected to be motivated to attend meetings, to develop trust, or even to adopt the technology when social pressures from a distance are weak? Even management and technical support for these teams at the local level may be weak: managers may consider such teams to be a part-time activity; local sites may lack gurus, champions, or even compatible hardware.

The topic of virtually colocated teams is distinguished from a general discussion of the problems involved in distributed work. The key word in this paper is teams, and the focus is on how the development and sustainability of social processes in the group are affected by the use of technology. The challenges that these teams face are directly tied to the limitations that the technology imposes on the communication of relevant social information, believed to be essential to the effectiveness of teams.

It is widely believed that a well-functioning team needs to forge common goals, working procedures, and rules of interaction (e.g., Weick 1969). Researchers are accumulating evidence that suggests that the ability of a distributed team to function depends on such factors, and not only on the capability of the technology to enable communication and data-exchange. The next section describes the role of interaction in developing perceptions of membership of a social/working group. Section 3 describes the methodology of the empirical study. Section 4 explains the challenges, illustrating them with examples found in virtual meeting interaction. The conclusion outlines further research directions.

\section{Interaction and Group Formation}

This paper discusses how limitations due to technology and distance make it difficult for geographically distributed teams to develop certain social processes in order to function as a "well-formed" team. However, a well-formed team, especially one that is geographically distributed, is hard to define. Originally Lewin (1948) proposed that a group becomes a social system when members' goals and their means to attain these goals become interdependent. Rabbie and Horwitz (1988) elaborate on this idea by adding that not only is the perceived interdependence of individual members essential to forming a group, but they must also experience a common fate. The notion of managing mutual 
interdependencies is a central idea that has been proposed to define cooperation in a group (Schmidt and Bannon 1992). However, an alternative view by Tajfel and Turner (1986) explains that members can still perceive themselves as a group even though their goals may not be interdependent. Rather, the process of emphasizing and defining social category differences defines a group for individuals.

Interaction would seem to facilitate perceptions of interdependence or a distinct social categorization for the group. Most investigations of group formation have been performed with groups who are physically colocated, i.e., whose primary contact is faceto-face. The value of interaction has been characterized as enabling individuals to slowly merge their attitudes and develop a new set of "group" attitudes, identifications, and behaviors, which becomes a collective, or congruent structure (e.g., Newcomb 1968; Weick 1969). Hackman (1976) describes that interaction and common stimuli play a major function for group members in influencing their affective and informational states. Interaction is especially critical in the initial stages of group formation. Its function has been explained to evaluate others' discrepant and conforming behaviors (Moreland and Levine 1989) and also to exchange information in order to seek commonalities (Tuckman and Jensen 1977).

With teams that are virtually colocated, it is not clear how interaction should be assessed. With the use of computer media, communication in groups changes along a number of dimensions, e.g., the overall amount increases (Hiltz et al. 1986), back-channel responses decrease (O’Conaill, Whittaker, and Wilbur 1993), and meetings last longer (Gallupe and McKeen 1990). Such changes certainly must alter the nature of interaction, but how they do is still largely unknown. Even though interactivity has been proposed as a mechanism responsible for promoting engagement, and possibly stable membership in on-line groups (Rafaeli and Sudweeks 1997), the nature of this interactivity still needs to be clarified. One aspect of interaction identified in a virtually colocated group is that the effectiveness of information exchange has been shown to correspond to the relational links of the members (Warkentin, Sayeed, and Hightower 1997). It does appear, though, that computer-mediated communication partners strive for clear interaction, as shown by their adoption of compensatory strategies to reduce confusion, at least in text-only systems (Herring 1999).

That interaction plays a role in shaping a group's culture, norms, cooperative attitude, and identity is hardly disputable. But now we enter an era where individuals are meeting from a distance and their interaction is both enabled and constrained by technological means. This paper attempts to define challenges for teams whose primary means of communication and operation is through technology. The claim put forward here is that group attributes are affected as a result of this communication, and the focus is specifically on participation, culture, and integration into other work.

\section{The Study}

Most of the arguments presented in this paper are based on an empirical study, performed in 1998, of virtually colocated teams done at The Boeing Company. Part of the results from this empirical study are reported in Mark, Grudin, and Poltrock. In 1996, two years before the study began, mergers with two other large corporations nearly doubled the 
number of employees in the organization and expanded their geographic distribution to the entire country. This redistribution led to the development of geographically distributed teams. The motivation of the study was to understand better the problems and benefits for such teams meeting regularly using desktop conferencing. The arguments in this paper are further developed with empirical results of other studies which examined synchronous communication among distributed actors and in distributed teams.

In the study, four geographically distributed teams of eight to 15 members, which had existed for six months or longer in the company, were observed during their weekly meetings for a three-month period. All teams used desktop conferencing technology combined with telephone conference calls to support their meetings. The desktop conferencing enabled application sharing. Most team members participated in the meeting from their desktop. In two of the groups, some participants gathered in a conference room, where a shared PC or a laptop connected to a projector enabled everyone to see. An ethnomethodological approach was used: for two groups, the observer regularly connected to the desktop conferencing meeting from her own desk; for the other two groups, the observer sat in the conference room where part of the team met face-to-face. The observer took notes (recording was not permitted) and supplemented the observations with on-line questionnaires, meeting agendas, minutes, chat, attendance records, and in-depth interviews conducted with key members of the teams.

The four teams included a Scientific Problem solving team, a voluntary team that solved real problems for the company; a Technical Working group, whose goal was devising standards for the company Web; a distributed Staff, whose manager was located in another city and whose team goal was information exchange; and a Best Practice team, with the goal of designing vehicles better, faster, and cheaper. Most meetings lasted between one and a half to three hours, and the meeting formats varied in the groups. In the first two teams, presentations (Power Point) were generally followed by discussion. The Staff mostly discussed agenda items, with an occasional presentation. The Best Practice team reviewed the status of action items assigned to team members and decided upon new ones.

\section{The Challenges}

Three different challenges were selected for virtually colocated teams. These three challenges are important for practitioners to consider in trying to design and implement appropriate technical support. All of the challenges that face such teams cannot be listed in this paper. Rather, key examples of team behaviors that are profoundly affected by computer-mediated interaction have been selected. The argument is that not only does technology use produce immediate behavioral effects in the group, but more importantly, these observable effects will have long-term consequences on the team development and effectiveness and, therefore, must be considered seriously. The challenges that will be discussed include: (1) achieving a high standard of participation, which affects impressions, interaction patterns, and trust; (2) developing an appropriate culture for high motivation and cooperation; and (3) integrating the remote team suitably into one s current working sphere. These challenges are not independent, but intricately related. For example, limited participation and discussion shapes the way that culture will be developed. 
The challenges exist at different levels of granularity. This is to be expected, as team work is composed of a combination of individual and group effort, amidst a background of organizational constraints. The participation challenge exists for individuals in the team. Social cues transmitted or restricted by the media, and clarity of the channel, affect individual perceptions. Developing a suitable culture to promote engagement, and motivation in the group, is a challenge at the team level. On the other hand, integrating work from a geographically remote team that is organizationally distant into one's current work is a challenge that may only be solvable through organizational change.

\subsection{The Participation Challenge}

Virtually colocated groups communicating in real time via audio and video conferencing face a definite challenge in making participation easy for members. The awkwardness of participation not only impedes the current discussion, but also results in long-term cumulative effects for the group. Several interaction phenomena observed with audio and video communication will be reviewed first.

Ruhleder and Jordan (1999) found that transmission delays in video conferencing negatively impacted group interaction by resulting in collisions, unnecessary rephrasing, and misapplied feedback. Even though video cues were available, these problems still occurred. The authors point out that when different actors hear different conversational segments due to delays, the actors' different expectations and interpretations inhibit the construction of a shared meaning.

In a study of an audio-only media space, Ackerman et al. found that interaction suffered from people not knowing who was present and that turn-taking was awkward. This finding was confirmed by Mark, Grudin, and Poltrock, who also found that actors had difficulties with identifying remote speakers. In the large distributed organization, actors often did not know to which organizations the speakers in their teams belonged. Difficulties in turn-taking were also observed by Bowers, Pycock, and O'Brien (1996) in audio communication in a collaborative virtual environment.

An example of the kind of awkwardness in turn-taking, and the problem of not knowing identities, or who is present, is shown here, from distributed teams in the current study. These kinds of exchanges are heard often during the team meetings:

Remote: I'd like to make a suggestion.

Leader: Is this Anita?

Remote: Yes.

M: $\quad$ Everyone clear so far? [One "yeah" is heard].

Dan: I have a quick question. Paul, are you still here?

$\mathrm{Al} \quad \quad$ Who is this speaking?

Carol: $\quad$ I'm Carol, I work at ..., my area is data exchange.

[long pause] 
Carol: Is everyone still there?

[a few say yes]

Carol: $\quad$ Because I didn't hear the background noises and didn't know if everyone is still there.

In face-to-face conversation, communication mechanisms exist that manage conversational flow through a rich and varied set of behavioral cues (Duncan 1972). These include intonation, gesture, gaze, and back-channel responses, which serve to turn conversation into a smooth dance among partners, without bumping into each other, so to speak.

It is questionable whether video images provide a real advantage for coordinating interaction. Isaacs and Tang (1993) found video advantageous for communicating nonverbal expression and reasons for pauses, yet found it disadvantageous for turn-taking and floor control. Similarly, Sellen (1995) found that turn-taking and other conversational aspects were not facilitated by adding video to audio. She concluded that there are no observable effects on turn-taking and synchronization when adding video to audio.

In the short term, these effects of the technology impact the group by limiting the richness of discussion. Isaacs et al. (1995) found that, in attending distributed video presentations, audience members were less likely to ask follow-up questions or clarifications, compared to face-to-face interactions. In the current study, it was discovered both from observations, and confirmed from interviews, that team members at remote sites find it hard to interject. Nonverbal cues serve for them as signals that a conversation turn has ended; without such cues, remote members prefer to err in favor of not speaking, and thus not interrupting.

However, few researchers have considered the long term impacts for a group in experiencing communication delays, awkwardness in turn-taking, not knowing who is present, who the speakers are, the meaning of pauses, and whether others are listening. In the long term, they create expectations for a certain type of interaction that can easily become a pattern for the group. It is not even clear whether distributed groups can develop norms to regulate conversation flow and establish positive interaction patterns. In longitudinal studies, Ackerman et al. found that group members developed norms to regulate their conversational exchange, but Mark, Grudin, and Poltrock and Ruhleder and Jordan did not, even among people who were experts in using the technology. One difference is that in the Ackerman et al. study, cooperation was continuous, among a welldefined set of team members. In the latter two studies, communication in the media space was discontinuous, with larger numbers of people. This may be more representative of the type of virtual meeting interaction that can be expected in large organizations.

Another type of consequence as a result of the awkwardness of participation is that it inhibits spontaneous behavior. Granted, in many face-to-face formal meetings, especially those run by strict meeting protocols or facilitation, spontaneity is not encouraged. In the teams studied at Boeing, however, during most parts of the meetings, free discussion was encouraged - for example, in posing questions to a presenter, in offering relevant information to the group, or in problem-solving discussions. Yet spontaneity was often not observed among the members and its absence was described in the interviews. 
Many of the team members in the study expressed in interviews that it was difficult to interpret others' on-line conversations. They remarked that facial expression helps to establish a context for them, to understand in which light they should frame a comment. Understanding the context in turn helps them to formulate a response (Clark and Schaeffer 1987). As the users described:

I get extra feedback of the body language of a person. Having met that person, I have that in the back of my mind [during a desktop conferencing session]. Without it, something is missing.

Reflective looks means they are thinking. Silence on the line doesn't. People may say things sarcastically, but the expression on-line is confused. Many signals that you have in face-to-face are lost.

The "sterile" type of interaction that forms in the group subsequently affects the potential for further group development. It creates expectations of the kinds of interaction possible in the group; for example, that after a ten-minute monologue, no one in the group may be listening. Even worse, the limitations on the interaction and richness of discussion can lead to the formation of stereotypes of other group members, as opposed to a more multi-faceted view of them. Research has shown that frequency and length of interaction is negatively associated with the reduction of bias toward others (Wilder and Thompson 1980) and interaction encourages partners to disconfirm stereotypes of each other (Cook 1985). This idea was recognized by a team member, who stated that face-to-face interaction helps him form a better model of the other:

It has value. When a person speaks, I attach a face and personality to him. When I hear a voice, I'm dying to associate the face with a voice. The only way to get a goodness of interaction is best to represent the person. You have to interact with this person. Your stereotype of the person doesn't work on the program, the person works on the program.

Additionally, "impolite" behaviors such as non-response, silence on the line, and interruptions are often attributed to the individual, and the role of the technology in contributing to this is downplayed. This phenomenon is known as the fundamental attribution error, the tendency for people to attribute the cause of behavior to an actor, rather than to situational factors (Ross 1977). This misattribution further impedes the development of the group as it can convey rudeness, or lack of involvement, as shown in this example:

Leader: Susan, anything else?

[long pause]

Leader: Susan left us awhile back.

Susan: No, I haven't. [Susan then explains that she put two URLs in the chat window. The leader replies that he did not have the desktop conferencing shared application running] 
The team leader did not see what Susan was doing, i.e., that she was actively participating in the meeting by putting URLs into the chat window. He had instead assumed that she had left the meeting.

Another example occurred when one member of the Scientific Team began drawing on the shared whiteboard of the desktop conferencing system. It was very slow and took time for the remote members to receive the image. The remote members commented that the Scientific Team members, who were located in the conference room, were excluding them from the discussion, when in fact they were watching the member draw on the whiteboard.

Another long-term consequence of the interaction difficulties is on the formation of trust in the group. Trust involves forming an expectation of reciprocation. Trust is explained by Gambetta (in Hinde and Groebel 1991, p. 5) as

a particular level of the subjective probability with which an agent assesses that another agent or group of agents will perform a particular action, both before he can monitor such action (or independently of his capacity ever to monitor it) and in a context in which it affects his own action.

Being able to assess or predict the actions of others involves learning about their behavior, preferably in as many varied situations as possible in order for the predictions to be robust. The lack of nonverbal behavior in computer-mediated communication, combined with inhibited participation, hinders people in developing robust predictions of others' behaviors. It is easy to understand how trust can be broken down in an audiomediated conversation. If a delay occurs in the transmission and the speaker does not hear a response, she may form an assumption about the others' reaction to her remark. If the speaker misspeaks, the facial expression is not visible in order for the speaker to repair the mistake (Duncan 1972).

Many of the team members expressed their intuitions about this relationship between their computer-mediated communication and building of trust:

You build relationships in face-to-face meeting. I notice that in the council time, the work in the group, and the social time, this all builds relationships. When you build trust with...top directors, then they are more likely to deal directly with you. It's easier to get through to them, and making contact with them is easier.

Trust is not a [desktop conferencing] issue. You build trust by working with the individual.

[Desktop conferencing] is possible only after trust is established. Problems are amplified by not meeting face-to-face. The real content of communication is not on the screen. If someone says something and then pauses, you can imagine anything. 
Everyone has a public and hidden agenda. You can't get hidden agendas from [desktop conferencing]. Here [face-to-face meeting] I can see everyone, look at the body language.

Until roles and activities are well-shared, people will be suspicious. As experience with team members grows, the predictability grows. For example, are people disclosing agendas that are not apparent?

On the other hand, a few members do not expect that the distributed team should develop a high level of trust. One member differentiates between a high level of trust which is necessary for his physically colocated team because he depends on them, and the virtually colocated team, which is "several organizational boundaries away." Another describes:

The level of trust that we have is appropriate for the group. It is not a close-knit team, as...a basketball team. Therefore, it is not so necessary.

Several members described that trust for them meant authenticating the knowledge of the others on the team, i.e., that they had competence in the context of their field. Keeping in mind that many of the members had not met each other and come from different organizations within the company, it is hard for them to judge the level of expertise and competence of others. Restricted verbal conversation is not sufficient for learning about others' knowledge.

\subsection{The Culture Challenge}

Groups, as Schein (1990) points out, can have their own cultures. Although different definitions of culture have been offered for groups and organizations (e.g., behavioral regularities-Goffman 1967) and the general atmosphere in which people interact (Tagiuri and Liwin 1968), Schein's definition of the shared assumptions and beliefs that are held by members of a group, that is a learned product of group experience is used here. Now when discussing distributed groups, we are faced with the problem of establishing what a definable group with a history means. There may be a roster with a list of members, but who actually attends scheduled meetings may be variable and even unknowable, especially when presence is not announced. Schein discusses the importance of having sufficient common experiences to have developed a shared view. This creates a challenge for the distributed group whose members themselves may not be able to define who is a member of their social unit. This is especially the case if attendance is not regular and if the group does not have a long history of meeting face-to-face.

On the other hand, assumptions of mission and operation can be formed implicitly, and patterns of behavior can set in quickly. It can be misleading to presuppose that teams, because they are geographically distributed and victim to some of the interaction difficulties described earlier, lack a culture. There is a danger of fooling ourselves if we believe the solution to effective teaming is simply that of presenting a set of recommenda- 
tions that the group can follow. By following these recommendations, the group can use the technology more efficiently and conduct better meetings. Wrong. We first have to discover whether a set of assumptions exists for the members in order to change them. And change, once culture is set in place, does not come easily.

Culture in a group is affected by a number of factors: the group's history, its experience, its structure, the larger organization, the composition of its members, and the environment. However, additionally in a virtually colocated group, the nature and usage of the technology also plays a major role in shaping the group's culture. Deep-seated assumptions in a group can be manifest through its handling of artifacts, its products or creations, or overt behavior of the group members, as Schein describes. In a virtually colocated group, when, due to the technology use, patterns set in of interruptions or lack of engagement in the meeting, although these are not favorable cultural manifestations, they are observable traits by which the group operates. They often reflect deeper assumptions by which the group functions. To illustrate this, the case of the Best Practice team is presented. The members, who had been meeting for about nine months, developed the behavior of multi-tasking during the weekly desktop conferencing meetings. The meeting format was to review action items. A spreadsheet scrolled down on the shared display and the team member responsible for the action item would report on its status: done or due date extended. The meeting had very little interaction among the members, influenced by the action item reporting practice. The members soon began to do multitasking until their action item was reached or until they were called upon to comment about the action item. The following meeting excerpt was quite typical for their interaction (see Mark, Grudin, and Poltrock 1999):

$\begin{array}{ll}\text { Mark: } & \text { Dan, that's your action item. } \\ \text { Dan: } & \text { Sorry, I didn't catch that. } \\ \text { Mark: } & \text { Jack, what's your comment? [long pause] Jack, are you there? } \\ \text { Jack: } & \text { I had my mute button on. } \\ \text { Mark } & \text { Next is the rotocraft area, but he is not here. } \\ \text { Rob: } & \text { We need communication. } 80 \text { hours is not the problem. I waste } 80 \\ & \text { hours talking on the telephone. It requires a tremendous amount } \\ & \text { of extra effort to clearly communicate. When someone says we're } \\ & \text { having a telecon, then we need to be on the telecon....People need } \\ & \text { to be on the telecon. We need a schedule. } \\ & \text { We all sit here and what are we doing? Is everyone trying to be on } \\ & \text { these telecons?....We have to decide on issues that we feel are } \\ \text { Joe: } & \text { important. Things can be done but we need to talk to each other } \\ & \text { and use these telecons. } \\ & \text { Thank you, Joe. [Mark moved on to the next action item] }\end{array}$

The multi-tasking resulted in a lack of engagement in the discussion and, thus, low commitment to the meeting. As is shown here, the members sometimes did express frustration, yet the group leader did not try to counteract the problem. One possible interpretation of the group's practice is that they lacked a culture. Instead it might be argued that the practice of multi-tasking during the meeting, and the corresponding lack of engagement, was the group culture. Contrary to the broader organizational culture, 
which promoted participatory decision making and where group meetings were expected to have high engagement and contributions of the members, the assumption of this group was that only minimal involvement was necessary during a virtual meeting.

Although multi-tasking occurred to some extent in other groups that were investigated, in no other group did the lack of engagement approach the level found in this group. This suggests that lack of engagement is not a behavior that is found in all virtual meetings. Multi-tasking was also reported by Isaacs et al. in a video broadcast setting; however, unlike a team setting, audiences would not necessarily be expected to be highly interactive with the speaker.

In fact, the members gained benefits by multi-tasking. Since their main task was reporting on the status of an action item, they needed only to be peripherally (or not at all) aware of others' reports in the meeting and could conduct other work in their offices in parallel. On some occasions, they even met with others in their offices during the virtual meeting. Yet overall, this group, who was expected to design a virtual colocation program within their division, did suffer by the lack of involvement. The meetings took more time because people had to refresh members about previous discussions. Information was unnecessarily repeated. Sometimes hostility was expressed by different members concerning lack of engagement and miscommunication. Since the action items were interrelated, directed toward achieving a common goal, learning about others' contributions could often have helped members with their own action items. Frequently the team leader asked people if they knew certain information; it was unclear whether the silence on the line was due to not knowing the answer or simply due to lack of involvement. For example:

Leader: Has everyone the information? [long pause] I assume silence means yes.

The team displayed very different types of overt behaviors when they met face-toface, in contrast to their virtual meetings. The different overt behaviors reflect different expectations that the members had for their face-to-face and for their virtual meetings. For example, in one face-to-face meeting, where members flew in from all over the U.S., the group used a facilitator. The purpose of the meeting was different than the typical virtual meetings of reviewing items; its goal was to prepare a charter. In contrast to the virtual meetings, the discussion was quite free, with many interjections that the facilitator had to cut off. There were jokes, clarifications, debate, nonverbal gesturing and facial expressions in response to comments in short, a high degree of interaction and engagement by the members.

One way that the technology may have influenced the interaction style is through the provision of a shared reference in the desktop conferencing; i.e., all members had the same view of the information. In the face-to-face meetings, members often referred back to points discussed earlier. Here we can see an influence of the technology: the shared application defines the focus, or view, for the group. In a face-to-face group, people are focused not only on the information, but on each other. (Also, the members in the virtual meeting may not have paid attention to earlier items, which could also explain why they did not refer back to points.) 
It might be argued that the multi-tasking, and corresponding lack of engagement, made the most sense for the group members, given the action item reporting format of the meeting. However, other models of interaction were possible in the group and the claim is that the group would have benefitted more had the members paid more attention at meetings. The argument is that the interaction style that the group adopted was shaped by different factors: the technology and the meeting format interacted with other factors, such as the history of the group and composition of the members.

If we examine the group's history, we find that, after the company merger, the team was formed officially by a vice president and assigned the goal of designing vehicles better, faster, and cheaper. The team was intentionally composed of representatives from all geographical areas of this company division, one person per site, spread across the entire United States. It is important to consider that the members stem from the different heritage companies and also different organizations within each of the former companies. The different members thus come from organizations that had different company cultures (e.g., participatory and tree-structured decision-making models) and different language codes (e.g., different acronyms). Since the group began, they had met about four times face-to-face. Given the members' differences, it is perhaps not surprising that this type of behavioral pattern set in. One team member even expressed that the meeting, and the technology use, was not being used to its full potential. A final product so far had not been created by the group.

Thus, developing a shared set of assumptions to promote positive group behaviors is a challenge for a distributed team. Culture forms from a number of influences and it is important to consider that the technology, and how it is used, comprise a key factor in shaping a distributed group's culture.

\subsection{The Integration Challenge}

The third challenge of virtually colocated teams is to discover ways that they can integrate remote team colleagues into their current working spheres. Work is continuous and complex. People move fluidly from one task to the next. The problem with virtually colocated teams is that the end of meetings often signal "out of sight, out of mind." Remote people and tasks from remote teams are thus not part of the continuity of each other's working spheres. Rather, as the team meetings in Boeing were, they fulfilled specific functions at specific points in time. In the words of the manager of the Staff, "it compromises the ability to get involved in things at the right time."

What does integration mean? It refers to more continual communication than just weekly or bimonthly formal meetings, with occasional telephone or e-mail exchanges in between. It refers to learning more about the other members on the team: their organizational homes, their expertise, their backgrounds, which currently is not the case in the teams studied. It refers to the exchange of results with selected team members to get feedback when the results are ready, instead of waiting for the formal meeting time. It refers to building a shared repository of common team materials, e.g., a shared workspace, accessible to all from remote locations. Again, currently none of the teams have such a shared space for team materials. Web sites exist, but it is not easy for team members to upload information. Integration refers to engaging in informal as well as 
formal communication, which can even lead to building relationships outside of the formal meeting structure. In all the interviews conducted, only one member from the Scientific Team reported contact with another remote team member outside of the formal meeting time, based on finding common interests. The Technical Working group used chat during the meetings, where informal communication took place. A member reported that it helped the team bond. It may in fact have served more a purpose of "breaking the ice" during the meeting than of bonding.

The lack of integration of the remote team work into each others' current working spheres became manifest in different ways in the organization. First, one consequence for the lack of integration is found with adoption of the technology. Remote team members had problems in adopting the desktop conferencing technology at Boeing (Mark, Grudin, and Poltrock 1999). These problems included downloading the software, securing the appropriate computers to run the software, and learning how to use the technology. One reason cited was that the team was viewed as a part-time activity and, therefore, assigned less importance than other tasks with people with whom one is colocated. This was also true of the members' local site managers. They viewed the team as part-time and were reluctant to fund appropriate equipment needed for the software.

Second, another consequence we might expect of the lack of integration is that members may not give high priority to the assigned tasks for the virtually colocated group. Signs of this were from the large numbers of action items in the Best Practice team that were delayed. However, a comparison of task completion in the members' face-toface group would need to be done to determine this.

\section{Discussion and Conclusions}

This paper described three challenges for teams with long-term agendas who are virtually colocated. Much previous research into the effects of computer-mediated communication in groups has focused on identifying the immediate communication difficulties that people experience. It is hoped that the challenges outlined in this paper can spark more longitudinal research into the effects of technology and distance on team building. Dissecting the process of team development identifies a number of social processes in the group, such as the development of participation patterns, culture, norms, refinement of roles and procedures, cohesion, identity, motivation to perform, trust, and leadership.

In considering team participation, not only does interaction via computer-mediated communication present immediate interaction difficulties, but it also has consequences on the long-term development of group processes. Not knowing who is present, not knowing identities or organizations, misattributions of behavior, inhibited participation from remote members, and the lack of nonverbal behavior to help interpret on-line speech, all affect the group development. I have argued that they contribute to expectations and patterns of certain participation behaviors, as well as impeding the building of trust.

Virtually colocated teams can have their own unique cultures, shaped by the use of the technology. The assumptions shared by the group members may be different for a group's virtual meetings with technology than a group's face-to-face meetings. In the case of the Best Practice team, the lack of engagement of the members was much higher than 
in the other teams observed. It seems reasonable to argue that the structured meeting format and shared reference of the desktop conferencing, combined with the group members' origins in different organizations, all contributed to the members' multi-tasking behaviors. Although it brought the members benefits, the price in the long-term was a set pattern where low commitment and lack of engagement in the meeting was accepted. Certainly multi-tasking brings advantages: it enables people to attend and monitor more meetings while working in parallel. Yet practitioners need to reflect on whether multitasking should be promoted for peripheral meeting participation rather than for people who are core group members.

All of the groups in the current study met at specific points in time for formally structured meetings. All of the groups had long-term agendas. Two groups had major tasks to accomplish for the company. Again we see a challenge that is really a paradox. Virtually colocated team members are expected to produce high quality deliverables on schedule. Yet group members limit their main involvement to the formal meetings. Many teams would in fact benefit from more continuous communication and involvement with each other and with the task. This is not to suggest more formal involvement, but rather more regular informal communication, which has been shown to provide value for teams (Whittaker et al. 1997).

The integration challenge applies at two levels. First, it addresses how people can seamlessly integrate their remote team activities into their current activities. Secondly, more broadly, it is a challenge to make the remote sites of the newly merged company more of a presence in the company. Ultimately, the goal would work toward creating one company culture.

The diversity of team members in virtually colocated teams increases the difficulty in integration. Team members stem from different organizational units. The organizational rewards are given at the individual level: members of cross-unit teams do not get salary reviews; members of local teams get salary reviews. Further, team members give distributed groups less priority than groups at their local sites. This was reflected in the interviews and also in people's reflections on the level of trust they expect among virtually colocated partners. These views are held not only by the team members, but also by their managers, many of whom were reluctant to provide funding for the equipment necessary to run the desktop conferencing software.

How can the improvement of technology make a difference?

There are a number of ways that technology and coaching efforts can support virtually colocated meetings and research is already underway in many areas. One method is to display nondisruptive signals on-line showing people's status in the meeting. Other methods involve setting new roles. The problems of not knowing who is present, who is speaking, turn-taking, and interpreting silence on the line can be alleviated through appropriate forms of facilitation, as Mark, Grudin, and Poltrock discovered. It is worth considering a role of "producer" for the team who can insure that members receive appropriate visual and audio information about others during a meeting. Success has already been reported with the use of producers in interactive television (Benford et al. 1999).

The challenges of developing a positive team culture, and integration, are somewhat related. Poltrock and Engelbeck (1997) articulate the requirement of supporting opportunistic interactions through a combination of e-mail, telephone, and awareness mechanisms. Synchronous and asynchronous awareness mechanisms can help inform team members of each others' current and past activities, can spark discussions, and provide them with a context for their own work (e.g., Fitzpatrick et al. 1999). Chat has 
been shown to have value in supporting informal communication in distributed teams (Bradner, Kellogg, and Erickson 1999; Fitzpatrick et al. 1999). Shared repositories of information for the group would be beneficial, such as a shared workspace, where all can enter and download material. In this way, members need not wait for the scheduled meeting to view the results. They can have the results at-hand when they are ready, and could even discuss them prior to the meeting. In fact, meetings consisting of action item reports may not even be necessary, if the action item status could be made available to all in a shared workspace. In this case, the meeting time could be used for other purposes, such as creating a final product, as one member proposed.

Research is called for to understand new roles necessary in such teams: for example, appropriate forms of leadership and facilitation. Although it is costly to create new roles, if communication and technology use becomes more efficient, then it becomes an overall gain. Research is also called for to understand the role of face-to-face meeting in distributed teams. Studies of group development suggest that the early phases of group formation is the critical time for interaction: at this time, information exchange, the accommodation of the group to the individual (and vice versa), and settling of roles takes place. However, in some cases it is impractical for geographically distributed teams to meet face-to-face. Research is needed to better define face-to-face schedules, such as when "booster shots" may be needed. The meeting itself may be restructured so that certain tasks can be better accomplished face-to-face, while other tasks, e.g., those having fewer dependencies among the members, can sufficiently be accomplished from a distance.

A number of other challenges for virtually colocated teams, such as developing leadership and facilitation, collective goals, coordination, and technology adoption and use, were not addressed in this paper. There is a long road ahead for such research. Technology is shrinking the world, but the understanding of how teams can adapt to the new technologies is not yet keeping pace.

\section{References}

Ackerman, M. S., Hindus, D., Mainwaring, S. D., and Starr, B. "Hanging on the 'Wire: A Field Study of an Audio-only Media Space," ACM Transactions on Computer-Human Interaction (4:1), 1997, pp. 39-66.

Benford, S., Greenhalgh, C., Craven, M., Walker, G., Regan, T., Morphett, J., Wyver, J., and Bowers, J. "Broadcasting On-line Social Interaction as Inhabited Television," in Proceedings of the Sixth European Conference on Computer-Supported Cooperative Work, S. Bødker, M. Kyng, and K. Schmidt (eds), Copenhagen, September 12-16, 1999, pp. 179-198.

Bowers, J., Pycock, J., and O'Brien, J. "Talk and Embodiment in Collaborative Virtual Environments." in Proceedings of CHI'6. New York: ACM Press, 1996.

Bradner, E., Kellogg, W. A., and Erickson, T. "The Adoption and Use of 'BABBLE': A Field Study of Chat in the Workplace," in Proceedings of the Sixth European Conference on Computer-Supported Cooperative Work, S. Bødker, M. Kyng, and K. Schmidt (eds), Copenhagen, September 12-16, 1999, pp. 139-158.

Clark, H. H., and Schaefer, E. F. "Collaborating on Contributions to Conversations," Language and Cognitive Processes (2:1), 1987, pp. 19-41.

Cook, W. W. "Experimenting on Social Issues: The Case of School Desegregation," American Psychologist (40), 1985, pp. 542-60.

Duncan, S. "Some Signals and Rules for Taking Speaking Turns in Conversation," Journal of Personality and Social Psychology (23:2), 1972, 283-292. 
Fitzpatrick, G., Mansfield, T., Kaplan, S., Arnold, D., Phelps, T., and Segall, B. “Augmenting the Workaday World with Elvin," in Proceedings of the Sixth European Conference on Computer-Supported Cooperative Work, S. Bødker, M. Kyng, and K. Schmidt (eds), Copenhagen September 12-16, 1999, pp. 431-450.

Gallupe, R. B., and McKeen, J. D. "Enhancing Computer-mediated Communication: An Experimental Investigation into the Use of a Group Decision Support System for Face-to-face versus Remote Meetings," Information and Management (18), 1990, pp. pp. 1-13.

Goffman, E. Interaction Ritual . Hawthorne, NY: Aldine, 1967.

Hackman, J. R. "Group Influences on Individuals," in Handbook of Industrial and Organizational Psychology, M. D. Dunnette (ed.). Chicago: Rand McNally., 1976.

Herring, S. "Interactional Coherence in CMC," Journal of Computer-Mediated Communication (4:4), June, 1999 (http://www.ascusc.org/jcmc/vol4/issue4/).

Hiltz, S. R., Johnson, K., and Turoff, M. "Experiments in Group Decision-making: Communication Process and Outcome in Face-to-face versus Computerized Conferences," Human Communication Research (13:2), 1986, pp. 225-252.

Hinde, R. A., and Groebel, J. Cooperation and Prosocial Behavior. Cambridge, UK: Cambridge University Press, 1991.

Isaacs, E. A., Morris, T., Rodriguez, T. K,. and Tang, J. C. “A Comparison of Face-to-face and Distributed Presentations," in Proceedings CHI'95 (Denver). New York: ACM Press, 1995, pp. 354-361.

Isaacs, E. A., and Tang, J. C. "What Video Can and Can't Do for Collaboration: A Case Study," Proceedings of the Multimedia, 1993. New York: ACM Press, 1993, pp. 199-205.

Lewin, K. Resolving Social Conflicts: Selected Papers on Group Dynamics. New York: Harper, 1948.

Mark, G., Grudin, J., and Poltrock, S. "Meeting at the Desktop: An Empirical Study of Virtually Colocated Teams," in Proceedings of the Sixth European Conference on Computer-Supported Cooperative Work, S. Bødker, M. Kyng, and K. Schmidt (eds), Copenhagen, September 1216, 1999, pp. 159-178.

Moreland, R. L., and Levine, J. M. "Newcomers and Oldtimers in Small Groups," in Psychology of Group Influence, P. Paulus (ed.). Hillsdale, NJ: Lawrence Erlbaum, 1989, pp. 143-186.

Newcomb, T.M. "Interpersonal Balance," in Theory of Cognitive Consistency: A Sourcebook, R. P. Abelson (ed.). Chicago: McNally, 1968, pp. 10-51.

O'Conaill, B., Whittaker, S., and Wilbur, S. "Conversations Over Video Conferences: An Evaluation of the Spoken Aspects of Video-mediated Communication," Human-Computer Interaction (8), 1993, pp. 389-428.

Poltrock, S. E., and Engelbeck, G. "Requirements for a Virtual Colocation Environment," in Proceedings of ACM Group '97 ( Phoenix, AZ, November 16-19). New York: ACM Press, 1997.

Rabbie, J. M., and Horwitz, M. "Categories versus Groups as Explanatory Concepts in Intergroup Relations," European Journal of Social Psychology (18), 1988, pp. 117-23.

Rafaeli, S., and Sudweeks, F. "Networked Interactivity," Journal of Computer-Mediated Communication (2:4), March 1997 (http://www.ascusc.org/jcmc/vol2/issue4/index.html).

Ross, L. "The Intuitive Psychologist and His Shortcomings: Distortions in the Attribution Process," in Advances in Experimental Social Psychology, Volume 10, L. Berkowietz (ed.). New York: Academic Press, 1977, pp. 173-219.

Ruhleder, K., and Jordan, B. "Meaning-making Across Remote Sites: How Delays in Transmission Affect Interaction," in Proceedings of the Sixth European Conference on Computer-Supported Cooperative Work, S. Bødker, M. Kyng, and K. Schmidt (eds), Copenhagen, September 12-16, 1999, pp. 411-429.

Schein, E. H. Organizational Culture and Leadership. San Francisco: Jossey-Bass, 1990. 
Schmidt, K., and Bannon, L. "Taking CSCW Seriously: Supporting Articulation Work," Computer Supported Cooperative Work (CSCW), An International Journal (1:1-2), 1992, pp. $7-40$.

Sellen, A. J. "Remote Conversations: The Effects of Mediating Talk with Technology," HumanComputer Interaction (10), 1995, pp. 401-444.

Sproull, L., and Kiesler, S. Connections: New Ways of Working in the Networked Organization. Cambridge, MA: MIT Press, 1991.

Tagiuri, R., and Litwin, G. H. (eds.). Organizational Climate: Exploration of a Concept. Boston: Division of Research, Harvard Graduate School of Business, 1968.

Tajfel, J., and Turner, J. C. "The Social Identity Theory of Intergroup Behavior," in Psychology of Intergroup Relations, S. Worchel and W. Austin (eds.). Chicago: Nelson-Hall, 1986, pp. 7-24.

Tuckman, B. W., and Jensen, M. A. C. "Stages of Small Ggroup Development Revisited," Group and Organizational Studies (2), 1977, pp. 419-427.

Warkentin, M. E., Sayeed, L., and Hightower, R. "Virtual Teams versus Face-to-face Teams: An Exploratory Study of a Web-based Conference System," Decision Sciences (28:4), Fall 1997, pp. 975-996.

Weick, K. E. The Social Psychology of Organizing. Reading, MA: Addison-Wesley, 1969.

Whittaker, S., Swanson, J., Kucan, J., and Sidner, C. "TeleNotes: Managing Lightweight Interactions in the Desktop," ACM Transactions on Computer-Human Interaction (4:2), 1997 , pp. 137-168.

Wilder, D. A., and Thompson, J. E. "Intergroup Contact with Independent Manipulations of Ingroup and Out-group Interaction," Journal of Personality and Social Psychology (38), 1980, pp. 589-603.

Zack, M. H. "Interactivity and Communication Mode Choice in Ongoing Management Groups," Information Systems Research (4:3), 1993, pp. 207-239.

\section{About the Author}

Gloria Mark is an assistant professor at the University of California, Irvine, in the Computing, Organizations, Policy, and Society (CORPS) group, in Information and Computer Science. She was previously at the German National Research Center for Information Technology (GMD) in Bonn, Germany. She has been active in the field of $\mathrm{CSCW}$, having investigated a variety of different types of technologies in use: electronic meeting rooms, collaborative hypermedia, shared work spaces, and collaborative virtual environments. Recently she was a visiting researcher at The Boeing Company, where she conducted a study of virtually colocated teams. Her research interests include virtual colocation technologies, and the cognitive and social aspects of technology use. Gloria can be reached by e-mail at gmark@ics.uci.edu. 